\title{
Zur Streifenstruktur der Kossel-Möllenstedtschen Elektroneninterferenzen mit konvergentem Bündel II
}

\author{
Von E. Fues und E. H. WAGNer \\ Aus dem Institut für theoretische und angewandte Physik der Technischen Hochschule Stuttgart \\ (Z. Naturforschg. 6 a , 79-84 [1951]; eingegangen am 30. November 1950)
}

Zusammenfassung siehe I. Mitteilung, diese Zeitschrift 6a, 1 [1951]

$\S 6$. Die Streifenstrukturder Interferenzflecke beim Zwei- und Dreistrahlfall

$\mathrm{D}$ ie Intensitätsverteilung des Zweistrahlfalls wurde schon ausführlich von verschiedenen Seiten diskutiert (vgl. die Zusammenfassung bei von $\mathrm{Laue}^{1}$ ), so daß sich ein näheres Eingehen auf diesen Punkt erübrigt. Wir wollen nur die Endformel in unseren Bezeichnungen noch einmal anschreiben, um den Vergleich mit den folgenden Formeln des Dreistrahlfalls zu erleichtern. Man erhält aus den Gleichungen von Teil I, $\S 4$, zusammen mit (I. 53) und (I. 50) die auch für $s_{1} \approx s_{i}$. gültige Darstellung:

$\left|\varphi_{i}^{\mathrm{Z}}\right|^{2}=\frac{1}{2} \frac{1}{u^{2}+1}\left\{1+\cos \left(\tau+2 c_{i} \sqrt{\left.u^{2}+1\right)}\right\}\right.$,

wobei $\quad u=\frac{s_{1}-s_{i}}{2\left|s_{i 1}\right|}, \quad c_{i}=\frac{k D}{2}\left|s_{i 1}\right|$ gesetzt ist.

Die Interferenzstreifen minimaler Intensität sind gegeben durch $\left(\pi+2 c_{i} \sqrt{u^{2}+1}=(2 m+1) \pi\right.$, der ganze Intensitätsverlauf hängt nur von der einen Koordinate $\boldsymbol{u}$ ab, auf deren Bedeutung wir später eingehen werden.

Zur Diskussion des Streifenverlaufs im Dreistrahlfall gehen wir von der Pendellösung (I. 54) aus, deren Absolutquadrat sich folgendermaßen schreiben läßt:

$$
\left|\varphi_{i}^{\mathrm{D}}\right|^{2}=\left|\psi_{1 i}^{*} \psi_{i i}\right|^{2}\left\{1+|A|^{2}+2|A| \cos \Gamma^{+}\right\} .
$$

$|A|$ und $\Gamma^{+}$bestimmen sich aus

$$
\begin{aligned}
|A| e^{i \Gamma^{+}}=e^{i a^{+}} & \left\{\operatorname{Im}\left(\frac{2 \psi_{11}^{*} \psi_{i 1}}{p_{1 i}^{*} \psi_{i i}}\right) \sin \beta^{+}-\cos \beta^{+}\right. \\
& \left.-i\left[1+\operatorname{Re}\left(\frac{2 \psi_{11}^{*} \psi_{i 1}}{y_{1 i}^{*} \psi_{i i}}\right)\right] \sin \beta^{+}\right\}
\end{aligned}
$$

$(\mathrm{Re}=$ Realteil, Im $=$ Imaginärteil $)$, wobei die $\mathrm{Ab}$ kürzungen

$$
\left.\begin{array}{l}
a_{-\infty}^{+}=\frac{k D}{2}\left(2 w_{i}^{+}-w_{1}^{+}-w_{k}^{+}\right) \\
\beta^{+}=\frac{k D}{2}\left(w_{1}^{+}-w_{k}^{+}\right)
\end{array}\right\}
$$

verwendet wurden. Bei der Herleitung von (2) und (3) wurde die erste Näherung der Orthogonalitätsrelation (I.50) $\sum_{p=1, i, k} \psi_{1 p}^{*} \psi_{i p}=0$ benutzt, um $\psi_{1 k}^{*} \psi_{i k}$ zu eliminieren; in die Gleichungen (2) bis (4) sind nun die Formeln von Teil I, $\S 4$, für $\psi_{p q}$ und $w_{p}$ einzusetzen.

Das Dreistrahlproblem wird aber nur unwesentlich komplizierter, wenn man neben der starken Kopplung der Strahlen 1 und $k$ auch noch die starke Kopplung der Strahlen 1 und $i$ in derselben Formel berücksichtigt. Wie man durch Vergleich von (I. 53) und (I. 54) feststellt, muß dazu in der Pendellösung kein weiteres Glied zugefügt werden. Diese gilt dann für $s_{1} \approx s_{i}$ und für $s_{1} \approx s_{k}$, aber nur für $\left|s_{i}-s_{k}\right| \geqslant s$. Man hat damit gleichzeitig ein sehr einfaches Beispiel für die Anwendung der Eigenwertdarstellung (I. 31) und den dazugehörigen Formeln (I. 34), (I. 37) und (I. 43) (mit $N=3$ ), die alle starken Zweistrahlkopplungen eines $\mathrm{N}$-Strahlproblems erfassen.

Wir wollen die etwas mühsame, aber nicht weiter schwierige Ausrechnung der Gln. (2) bis (4) nicht vorführen. Berücksichtigt man dabei, daß wir uns auf solche Einfallsrichtungen beschränken wollen, für die stets $\left|s_{i}-s_{k}\right| \gg s$ und entweder $\left|s_{1}-s_{k}\right| \gg s$ oder $\left|s_{1}-s_{i}\right| \gg s$ ist, dann erhält man für die in (2), (3) und (4) auftretenden Größen

$$
\left|\psi_{1 i}^{*} y_{i i}\right|^{2}=\frac{1}{4} \frac{1}{u^{2}+1},
$$

$$
|A| e^{i I^{+}}=\frac{e^{i a^{+}}}{\sqrt{v^{2}+1}}\left\{a \sin \sigma \sin \beta^{+}-\sqrt{v^{2}+1} \cos \beta^{+}+i[v-a \cos \sigma] \sin \beta^{+}\right\},
$$

1 L. von Laue, Materiewellen und ihre Interferenzen, 2. Aufl., Leipzig 1948. 


$$
\left.\begin{array}{l}
a^{+}=-\frac{1}{2} c_{i}\left(u+3 \sqrt{\left.u^{2}+1\right)}+c_{k} v,\right. \\
\beta^{+}=c_{k} \sqrt{v^{2}+1}+\frac{1}{2} c_{i}\left(\sqrt{u^{2}+1}-u\right) .
\end{array}\right\}
$$

Die benutzten Abkürzungen haben folgende Bedeutung

$u=\frac{s_{1}-s_{i}}{2\left|s_{i 1}\right|}, \quad v=\frac{s_{1}-s_{k}}{2\left|s_{k 1}\right|}, \quad a=\left|\frac{s_{i k}}{s_{i 1}}\right|$,

$\left.c_{i}=\frac{k D}{2}\left|s_{i 1}\right|, c_{k}=\frac{k D}{2}\left|s_{k 1}\right|, \quad \sigma=\sigma_{1 i}+\sigma_{i k}+\sigma_{k 1} \cdot\right\}$

Hierbei ist

$$
\mathrm{s}_{p q}=\left|\mathrm{s}_{p q}\right| e^{i \sigma_{p q}}
$$

gesetzt, nach (I. 16) ist also

$$
\sigma_{p q}=-\sigma_{q p} .
$$

$u$ und $c_{i}$ charakterisieren das Zweistrahlproblem (1 $i$ ), $v$ und $c_{k}$ das Zweistrahlproblem ( $1 k$ ), wie man durch Vergleich mit (1) feststellt. Durch die Gln. (2) bis (8) wird der Intensitätsverlauf des Dreistrahlproblems bei Doppelanregung vollständig beschrieben. Die Tan-

gentialkomponenten des Ausbreitungsvektors $\mathfrak{f}_{i}{ }^{\mathrm{t}}$, die ja gleichzeitig als Filmkoordinaten die Intensitätsverteilung des Flecks $i$ beschreiben, treten in diesen Formeln nur noch in den beiden Variablen $u$ und $v$ auf, deren Bedeutung wir uns jetzt klarmachen wollen.

Dazu berechnen wir aus (I. 16)

$$
\begin{aligned}
s_{p}-s_{q}=\frac{1}{k^{2}}\left[\left(2 \mathfrak{f}_{1}{ }^{\mathrm{t}}+\mathfrak{h}_{p}{ }^{\mathrm{t}}+\mathfrak{h}_{q}{ }^{\mathrm{t}}\right)\right. & \left(\mathfrak{h}_{p}{ }^{\mathrm{t}}-\mathfrak{h}_{q}{ }^{\mathrm{t}}\right) \\
& \left.+2 k\left(\bar{h}_{p}^{\mathrm{n}}-h_{q}^{\mathrm{n}}\right)\right] .
\end{aligned}
$$

Diese Ausdrücke stellen also im $\mathfrak{f}$-Raum Parallelebenen zur Normalenrichtung $\mathfrak{n}$ dar. In der Filmebene kann $s_{p}-s_{q}=$ const als Geradenschar senkrecht zum Vektor $(d / k)\left(\mathfrak{h}_{p}{ }^{\mathrm{t}}-\mathfrak{h}_{q}{ }^{\mathrm{t}}\right)$ aufgefaßt werden ( $d=$ Filmabstand). Die Variablen $u$ und $v$ entsprechen also einem im allgemeinen schiefwinkligen Parallelkoordinatensystem in der Filmebene mit verschiedenen Achseneinheiten. Anstatt $(d / k) \mathfrak{f}_{i}{ }^{t}$ vom F'ilmursprung (Durchstoßpunkt der Normalen $\mathfrak{n}$ ) aus abzutragen, können wir zur Beschreibung des Flecks $i$ natürlich auch wegen $\mathfrak{f}_{i}{ }^{\mathrm{t}}=\mathfrak{f}_{1}{ }^{\mathrm{t}}+\mathfrak{h}_{i} \mathrm{t}$ den Vektor $(d / k) \mathfrak{f}_{1}{ }^{t}$ vom Endpunkt des Vektors $(d / k) \mathfrak{h}_{i}{ }^{t}$ aus abtragen. Da nun

$$
u=\frac{s_{1}-s_{i}}{2\left|s_{i 1}\right|}=\frac{1}{k^{2}\left|s_{i 1}\right|}\left(\frac{k}{d}\right)^{2}\left\{\left(\frac{d}{k} \mathfrak{k}_{1}^{\mathrm{t}}+\frac{1}{2} \frac{d}{k} \mathfrak{h}_{i}^{\mathrm{t}}\right) \cdot\left(-\frac{d}{k} \mathfrak{h}_{i}^{\mathrm{t}}\right)-d \frac{d}{k} h_{i}^{\mathrm{n}}\right\},
$$

so wächst $u$ in Richtung des Vektors $-(d / k) \mathfrak{h}_{i}{ }^{\mathrm{t}}$, bei verschwindender Normalkomponente $h_{i}{ }^{\mathrm{n}}$ halbiert $u=0$ den vom Endpunkt von $(d / k) \mathfrak{h}_{i}{ }^{\mathrm{t}}$ aus abgetragenen Vektor $-(d / k) \mathfrak{h}_{i}{ }^{t}$ [und damit natürlich auch den vom Filmursprung aus abgetragenen Vektor $\left.+(d / k) \mathfrak{h}_{i}{ }^{\dagger}\right]$. Gegen das Mittellot auf $(d / k) \mathfrak{h}_{i}{ }^{\mathrm{t}}$ verschiebt sich die

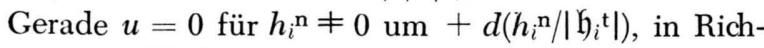
tung $-(d / k) \mathfrak{h}_{i}$ t. Ebenso wächst $v$ in Richtung von $-(d / k) \mathfrak{h}_{k}{ }^{\mathrm{t}} ; v=0$ ist für $h_{k}{ }^{\mathrm{n}}=0$ das Mittellot auf dem vom Endpunkt des Vektors $(d / k) \mathfrak{h}_{i}{ }^{\mathrm{t}}$ aus abgetragenen Vektor $-(d / k) \mathfrak{h}_{k}{ }^{\mathrm{t}}$ und verschiebt sich für $h_{k} \mathrm{n} \neq 0$ um $+d\left(h_{k}{ }^{\mathrm{n}} /\left|\mathfrak{h}_{k}{ }^{\mathrm{t}}\right|\right)$ in Richtung $-(d / k) \mathfrak{h}_{k}^{\mathrm{t}}$ gegen das Mittellot. Die Einheit für $u$ ist, wie ersichtlich,

$$
e_{i}=\left(\frac{d}{k}\right)^{2} \frac{k^{2}\left|s_{i 1}\right|}{\left|(d / k) \mathfrak{h}_{i}{ }^{t}\right|}
$$

und ganz analog für $v$.

Da $u=0$ mit der K i k u ch i - Linie $\mathfrak{h}_{i}$ zusammenfällt, hat man damit die Möglichkeit, den Durchstoßpunkt der Plättchennormalen $\mathfrak{t t}$ mit dem Film festzulegen. Man hat nur für zwei Reflexe, $i$ und $j$, Parallelen zu den Kikuchi-Linien $\mathfrak{h}_{i}$ und $\mathfrak{h}_{j}$ mit den Abständen $(d / 2 k)\left|\mathfrak{h}_{i}{ }^{\dagger}\right|-d\left(h_{i}{ }^{\mathrm{n}} /\left|\mathfrak{h}_{i}{ }^{\dagger}\right|\right)$ und $(d / 2 k)\left|\mathfrak{h}_{j} \mathrm{t}^{\mathrm{t}}\right|$ $-d\left(h_{j}{ }^{\mathrm{n}} /\left|\mathfrak{h}_{j}{ }^{\mathrm{t}}\right|\right)$ zu ziehen, diese schneiden sich dann im Filmursprung. Bei Glimmer benutzt man zur Orien* tierung auf den Aufnahmen üblicherweise den „Stern“ der Kikuchi-Bänder 060, 331 und $3 \overline{3} 1$. Der Durchstoßpunkt der Plättchennormalen liegt dann auf der Bandmitte von 060 (die Normalkomponente von 060 verschwindet) und ist um

$$
\frac{d}{\cos 30^{\circ}} \frac{h_{331}^{\mathrm{n}}}{\left|\mathfrak{h}_{331}^{\mathrm{t}}\right|}=0,0064 \cdot d
$$

in Richtung des Vektors (602) (also längs der Bandmitte von 060$)$ verschoben.

Die bisherigen Rechnungen und Überlegungen galten alle für Doppelanregung. Um die Gleichungen auf Umweganregung zu übertragen, müssen wir nun die Transformation (I. 56) auf unsere Gleichungen anwenden. Durch Indizesvertauschung in den Bezeichnungen (8) erhält man, wenn man die neuen Bezeichnungen durch Striche kenntlich macht,

$$
v^{\prime}=\frac{s_{i}-s_{k}}{2\left|s_{k i}\right|}, \quad c_{k}^{\prime}=\frac{k D}{2}\left|s_{k i}\right|, \quad a^{\prime}=\left|\frac{s_{1 k}}{s_{1 i}}\right| ;
$$


diese drei Abkürzungen müssen, dem anderen Problem entsprechend, von (8) verschieden sein. Dagegen wollen wir statt

$$
\begin{gathered}
u^{\prime}=\frac{s_{i}-s_{1}}{2\left|s_{1 i}\right|}=-u, \quad c_{i}^{\prime}=\frac{k D}{2}\left|s_{i 1}\right|=c_{i} \\
\sigma^{\prime}=\sigma_{i 1}+\sigma_{1 k}+\sigma_{k i}=-\sigma
\end{gathered}
$$

die alten Bezeichnungen $u, c_{i}$ und $\sigma$ weiter benutzen, um den Unterschied zwischen Doppel- und Umweganregung klarer hervortreten zu lassen. Dann bewirkt aber die Transformation (I. 56) in den Gln. (5) ff. nur ein Ersetzen von $v, c_{k}$ und $a$ durch $v^{\prime}, c^{\prime}{ }_{k}$ und $a^{\prime}$ und eine Vorzeichenänderung von $u$, da sich die Vorzeichenänderung von $\sin \sigma$ mit der von $\sin \beta^{+}$kompensiert. Außerdem geht Gl. (6) durch die Vorzeichenänderung der übrigen gekreuzten Größen in die konjugiert komplexe über, was natürlich für die weitere Rechnung vollkommen bedeutungslos ist. $v^{\prime}$ wächst in Richtung des Yektors $(d / k)\left(\mathfrak{h}_{i}-\mathfrak{h}_{k}\right), v^{\prime}=0$ ist für $h_{i}{ }^{\mathrm{n}}-h_{k^{\mathrm{n}}}=0$ das Mittellot auf dem vom Filmursprung aus abgetragenen Vektor $+(d / k)\left(\mathfrak{h}_{i}-\mathfrak{h}_{k}\right)$ und verschiebt sich für $h_{i}{ }^{\mathrm{n}}-h_{k}{ }^{\mathrm{n}} \neq 0$ um d $\left(h_{i}{ }^{\mathrm{n}}-h_{k}{ }^{\mathrm{n}}\right) /\left|\mathfrak{h}_{i} \mathrm{t}-\mathfrak{h}_{k}{ }^{\mathrm{t}}\right|$ gegen das Mittellot.

Wir wenden uns nun der Untersuchung des Interferenzstreifenverlaufs bei Doppelanregung zu. Dabei ist der Fall der Umweganregung immer in dem Sinn enthalten, daß man in allen Gleichungen $\boldsymbol{u}_{\mathbf{6}}$ durch $-u$ zu ersetzen hat und die Definitionen für $v^{\prime}, c^{\prime}{ }_{k}$ und $a^{\prime}$ berücksichtigt. Wir werden im folgenden auch die Kreuze wieder weglassen, da sie durch die vorangehenden Erörterungen überflüssig geworden sind.

Analog zum Zweistrahlfall erhält man beim Dreistrahlfall Interferenzkurven minimaler Intensität für

$$
I=(2 m+1) \pi .
$$

Nach Voraussetzung muß nämlich entweder $u$ oder $v$ grcß gegen 1 sein. Für $v \gg 1$ geht aber (2) in (1) über. Für $u \gg 1$ wird $\left|\psi^{*}{ }_{1 i} \psi_{i i}\right|$ und $|A|$ in (2) mit $u$ langsam veränderlich, wie man aus (5), (6) und (7) erkennt.

Man gewinnt also den Interferenzstreifenverlauf durch Diskussion des komplexen Arguments $T$ von (6). Diese wird dadurch etwas erschwert, daß die Wurzeln für $v=0$ eine Unstetigkeitsstelle haben. Da diese Untersuchung vom physikalischen Standpunkt weniger interessant ist, können wir uns darauf beschränken, die Ergebnisse mitzuteilen.

Das komplexe Argument $\Gamma$ von (6) wird

$$
I=\pi+a+\operatorname{arcctg} \frac{a \sin \sigma-\sqrt{v^{2}+1 \operatorname{ctg} \beta}}{v-a \cos \sigma} .
$$

2 E. Fue s, Z. Physik 125, 531 [1949].
Hierbei ist die Bestimmung des arc ctg für alle in dem Streifen zwischen $v=0$ und $v=a \cos \sigma$ gelegenen Punkte der $u v$-Ebene im selben Zweig der arc ctg-Funktion wie $+\rho$, für alle übrigen Punkte der $u v$-Ebene im selben Zweig wie $-\beta$ zu wählen.

Um den Verlauf der Interferenzstreifen (15) für $\Gamma=(2 m+1) \pi$ besser übersehen zu können, ist es zweckmäßig, sich zunächst deren Verlauf für sehr große $u$ klar zu machen. Dann reduziert sich Gl. (7) auf

$$
a=-2 c_{i} u+c_{k} v, \quad \beta=c_{k} l v^{2}+1
$$

und damit wird (15)

$$
\begin{aligned}
I= & \pi-2 c_{i} u+c_{k} v \\
& +\operatorname{arcctg} \frac{a \sin \sigma-\sqrt{v^{2}+1 \operatorname{ctg} c_{k} \sqrt{v^{2}+1}}}{v-a \cos \sigma} .
\end{aligned}
$$

Wendet man diese Gleichung auf den von $\mathrm{F}$ u e s ${ }^{2}$ behandelten Fall von Umweganregung bei Glimmeraufnahmen an, so erhält man (abgesehen von etwas anderen Bezeichnungen) bis auf Glieder höherer Ordnung genau die dort für das Cosinusargument angegebene Gleichung.

Die Kurven (17) sind nun nach $c_{i} u$ auflösbar

$$
\begin{aligned}
c_{i} u= & \frac{1}{2}\left\{-I+\tau+c_{k} v\right. \\
& \left.+\operatorname{arcctg} \frac{a \sin \sigma-\sqrt{v^{2}+1 \operatorname{ctg} c_{k} \sqrt{v^{2}+1}}}{v-a \cos \sigma}\right\}
\end{aligned}
$$

und verlaufen „im Mittel“ ebenso wie

$$
c_{i} \bar{u}=\frac{1}{2}\left\{-I+\pi+c_{k} v \mp c_{k} l v^{2}+1\right\}
$$

für denselben Wert von $\Gamma$. Dabei gilt das Pluszeichen vor der Wurzel nur in dem Streifen $(0, a \cos \sigma)$, sonst

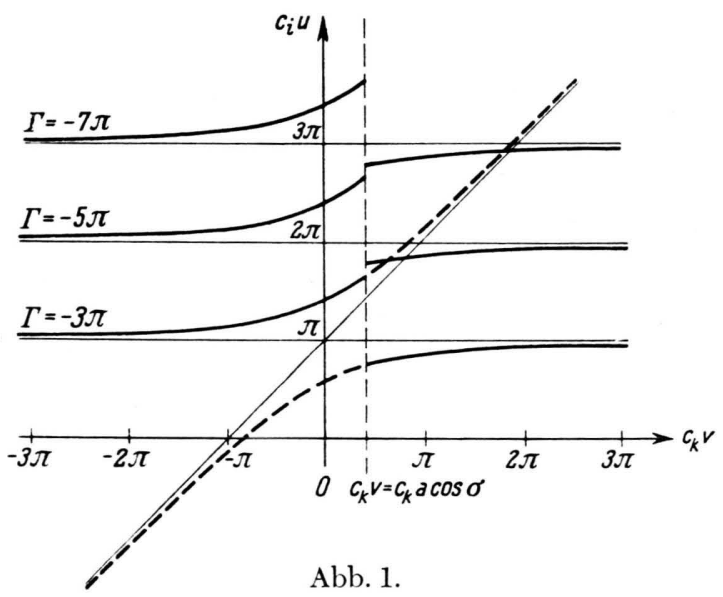


immer das Minuszeichen in dem durch Gl. (I. 30) erklärten Sinn. (19) stellt Äste einer Hyperbelschar mit dem Parameter $\Gamma$ dar, die in Abb. 1 in einem rechtwinkligen Koordinatensystem gezeichnet sind. Die Kurven $c_{i} \bar{u}(v)$ haben bei $v=a \cos \sigma$ eine Unstetigkeitsstelle und gehen für große $v$ gegen die Asymptoten

$$
c_{i} u=-\frac{I-\pi}{2}=-m \cdot \pi,
$$

wenn dort (19) noch gültig ist. Diese Asymptoten sind gerade die Kurven minimaler Intensität des Zweistrahlproblems ( 1 i) für $u \gg 1$, die aber natürlich für große $v$ wieder durch ein anderes Dreistrahlproblem gestört werden können, so daß diese asymptotische Annäherung gar nicht in Erscheinung tritt.

Um diese Kurven mittleren Verhaltens schwanken nun die Interferenzkurven $c_{i} u$ nach (18) mit einer Amplitude, die nur in gewissen singulären Fällen für $v=a \cos \sigma$ den Wert $\pi$ annehmen kann, für große $v$ jedoch sehr rasch abnimmt*.

Für $\beta=m \pi$ und an einer weiteren Zwischenstelle in jedem Zweig der arc ctg-Funktion, die für große $v$ gegen $\beta=\left(m+\frac{1}{2}\right) \pi-\sigma$ strebt, stimmen die Kurven $c_{i} u$ und $c_{i} \bar{u}$ genau überein. Der Abstand dieser Punkte (also die räumliche Schwankungsfrequenz) wird im wesentlichen durch die Konstante $c_{k}$ bestimmt. Diese Aussagen über die Schwankung der Interferenzkurven gelten auch für beliebige $u, \mathrm{~d}$. h. für die Gl. (15). Auch für (15) lassen sich analoge Kurven mittleren Verhaltens angeben, nämlich

$$
\left.\begin{array}{l}
I=\pi+a-\beta \quad \text { außerhalb des Streifens }(0, a \cos \sigma) \\
I=\pi+a+\beta \text { im Streifen }(0, a \cos \sigma) .
\end{array}\right\}
$$

Diese Kurven haben nun zwei Unstetigkeitsstellen. Man kann sie sich aus den in Abb. 3 gezeichneten Hyperbelästen entstanden denken, indem man die Ordinate dieser Figur ungleichmäßig dehnt. Diese Verzerrung wäre außerhalb des Streifens $(0, a)(\cos \sigma)$ um den Betrag $\sqrt{u^{2}+1} / u$ und in diesem Streifen um $\frac{1}{2}\left(u+\sqrt{u^{2}+1}\right) / u$ vorzunehmen. Die Asymptoten dieser Kurven sind die Interferenzkurven des Zweistrahlproblems nach Gl. (1), die nun nicht mehr äquidistant sind. Wegen der oben erwähnten Störung durch weitere Dreistrahlprobleme bleibt der Abstand der Interferenzkurven von den Asymptoten nicht nur in der engeren Umgebung von $v=a \cos \sigma$ merklich. Diese Er- scheinung soll künftig (vgl. die Fußnote S. 4 im Teil I) als Streifenverschiebung bezeichnet werden.

Die zweifellos interessanteste Eigenschaft der Interferenzkurven (15) bzw. (18) ist die Erscheinung der Streifenverbiegung. Wenn in (15) bzw. (18) $\Gamma=(2 m+1) \pi$ gesetzt wird, sprechen wir von einem Interferenzstreifen $m$-ter Ordnung.

Ein solcher Interferenzstreifen ist ein im mathematischen Sinn stetiger Kurvenzug $u=u(v)$, ausgenommen evtl. die Stellen $v=0$ und $v=a \cos \sigma$. Die Kurven (18) sind an der Stelle $v=0$ stetig. Die Kurven (15) haben dort dagegen eine "Unstetigkeit höherer Ordnung“ in folgendem Sinn: Nach Gl. (I. 30) müßten die Grenzwerte von $\beta=\beta(u, v)$ nach (7) der Gleichung

genügen.

$$
\beta(v+0, u)=-\beta(v-0, u)
$$

Das erste Glied von $\beta$ nach (7) genügt zwar dieser Gleichung, nicht aber das zweite. Dieses wird jedoch nach Voraussetzung $\simeq s^{2}$ für $v \rightarrow 0$ und könnte daher vernachlässigt werden, wenn es nicht in der Umgebung von $\boldsymbol{u}=\mathbf{0}$ in die erste nichtverschwindende Größenordnung von $\beta$ aufrücken würde. Wir müssen es deshalb beibehalten, da ja unsere Formel für $v \approx 0$ und für $u \approx 0$ gültig sein soll (nicht dagegen natürlich für $v \approx u \approx 0$ !) und dafür die durch dieses Glied hervorgerufene „Unstetigkeit höherer Ordnung“ der Interferenzstreifen an dieser Stelle in Kauf nehmen.

An der Stelle $v=a \cos \sigma$ kann der Streifen $m$-ter Ordnung dagegen eine echte Sprungstelle haben. Der Sprung des Funktionswertes $c_{i} u$ beträgt aber (wenn man von sirfoulären Fällen absieht, auf die wir später eingehen wollen) stets ein ganzes Vielfaches von $\pi$, d. h. eine ganze Zahl von Streifenabständen. Es ist stets (der Index $m$ soll die Ordnung des Interferenzstreifens andeuten)

$$
\begin{aligned}
c_{i} u_{m}(a \cos \sigma+0)-c_{i} u_{m}(a \cos \sigma-0) & \\
& =-l_{\pi}(l \geq 0) .
\end{aligned}
$$

Damit besteht aber das Interferenzstreifensystem als Ganzes aus stetigen Kurven; ein Streifen der Ordnung $m_{+}$im Gebiet $v>a \cos \sigma$ geht an der Stelle $v=a \cos \sigma$ stetig in den Streifen der Ordnung $m_{-}$im Gebiet $v<a \cos \sigma$ über.

Die Differenz $m_{-}-m_{+}$der Ordnung zweier stetig ineinander übergehender Interferenzstreifen springt immer dann um Eins, wenn $A \exp i \Gamma$ nach (6) verschwindet, was nur für $v=a \cos \sigma$ möglich ist. $A \exp i \Gamma$ verschwindet aber immer dann, wenn $c_{k}$ der Gleichung

$$
\hat{c}_{k}=\frac{1}{ \pm \mid \sqrt{a^{2} \cos ^{2} \sigma+1 \mid}}\left\{\operatorname{arcctg} \frac{a \sin \sigma}{ \pm|| a^{2} \cos ^{2} \sigma+1 \mid}-\frac{1}{2} c_{\mathrm{i}}\left(\sqrt{u^{2}+1}-u\right)\right\}
$$

* Man vergleiche zur Illustration die bei $\mathrm{F}$ u e s 2 gezeichneten Kurven, Abb. 3 bis 7 dieser Arbeit! 
genügt (dabei steht das obere Zeichen für $a \cos \sigma>0$, das untere für $a \cos \sigma<0$ ); oder in unserer Näherung

$$
\hat{c}_{k}=\frac{1}{\sqrt{a^{2} \cos ^{2} \sigma+1}} \operatorname{arc} \operatorname{ctg} \frac{a \sin \sigma}{\mid \sqrt{a^{2} \cos ^{2} \sigma+1 \mid}} .
$$

Die Konstante $c_{k}$ ist nach ihrer Definition stets positiv. Setzt man nun $\hat{c}_{k}^{(0)}=0$ und ordnet die sich aus (25) mit allen möglichen Bestimmungen der arc ctg-Funktion ergebenden positiven $\hat{c}_{k}$-Werte der Größe nach an, wobei also $0=\hat{c}_{k}{ }^{(0)}<\hat{c}_{k}^{(1)}<\hat{c}_{k}^{(2)}<\ldots$ sein soll, so gilt:

Die Differenz der Ordnung zweier an der Stelle $v=a \cos \sigma$ stetig zusammenschließender Streifen beträgt

$$
m_{-}-m_{+}=l>0,
$$

wenn

$$
\hat{c}^{(1)}<c_{k}<\hat{c}_{k}^{(1+1)} .
$$

Der Wert der „kritischen Konstanten“ $\hat{c} k$ ist zwar in unserer Näherung nach (25) nur von den Kristalleigenschaften $a, \sigma$ bestimmt. Wie man aber an dem beim Übergang von (24) zu (25) weggelassenen Glied höherer Ordnung erkennt, würde $\hat{c}_{k}$ in nächster Näherung auch noch von der Einfallsrichtung abhängig werden. Es ist deshalb durchaus möglich, daß die Differenz $m_{m}-m_{+}$für Streifen verschiedener Ordnung im selben Interferenzfleck längs einer „Verschiebungslinie“ $v=a \cos \sigma$ verschieden ist. Eine experimentelle Bestätigung dieser Aussage der Theorie wäre natürlich sehr erwünscht. Allerdings scheint es erfahrungsgemäß nicht ganz einfach zu sein, die Differenz $m_{-}-m_{+}$gerade in zweifelhaften Fällen aus den Aufnahmen zu bestimmen.

Die vorher erwähnten „singulären Fälle“ treten nun dann ein, wenn $c_{k}$ gerade gleich einem der kritischen Werte $\hat{c}_{k}{ }^{(1)}$ wird. Dann würde an der Stelle $v=a \cos \sigma$ tatsächlich eine Unstetigkeit im Interferenzstreifensystem auftreten, bei der sich ein Streifen minimaler Intensität in einem Streifen maximaler Intensität fortsetzt. Ein solches Verhalten wäre jedoch höchstens für einen bestimmten Streifen eines Streifensystems zu erwarten wegen der erwähnten Abhängigkeit von der Einfallsrichtung bei der nächsten Näherung von $c_{k}$. Es erscheint daher zweifelhaft, ob diese singulären Fälle überhaupt eine physikalische Bedeutung haben.

\section{§7. Zur Anwendung der Theorie}

In Teil I und dem vorhergehenden Paragraphen haben wir den allgemeinen Formalismus der dynamischen Theorie für den Laue-Fall des $\mathrm{N}$-Strahlproblems entwickelt, soweit sich dieser durch gleich- zeitige starke Kopplung von nicht mehr als zwei Strahlen erfassen läßt. Wir wollen nun die Frage erörtern, in welchem Umfang die eingeführten Voraussetzungen bei Glimmer als dem wichtigsten Aufnahmematerial erfüllt sind und abschätzen, welche Genauigkeit von den Aussagen der Theorie zu erwarten ist. Hinsichtlich der Streifenstruktur wollen wir uns dabei auf die wichtigsten und auffallendsten Glimmerreflexe 060, 331, $3 \overline{3} 1$ usw. beschränken, die durch ihre Größe, Deutlichkeit der Streifenstruktur und die fast stets auftretende Erscheinung der Streifenverbiegung besonders ausgezeichnet und interessant sind.

Wir können auch darauf verzichten, noch weitere Beispiele des Streifenverlaufs in einzelnen Reflexen bestimmter Aufnahmen auszurechnen und aufzuzeichnen. Einmal ist durch die Arbeit von $\mathrm{Fues}{ }^{2}$ schon gezeigt, daß dies tatsächlich in befriedigender Ubereinstimmung mit dem Experiment möglich ist. Außerdem soll in einer weiteren Arbeit eine (zwar nur qualitativ arbeitende) Methode dargestellt werden, die eine äußerst einfache Ermittlung der Streifenstruktur eines ganzen Reflexes aus der Ausbreitungsfläche des $N$-Strahlproblems ermöglicht [unsere Gln. (15) und (18) gelten ja immer nur für bestimmte Bereiche eines Reflexes]. Diese Methode soll auf eine ganze Reihe von Aufnahmen angewandt werden.

Wir wollen uns zunächst überlegen, welche Fehler wir machen, wenn wir in der Umgebung einer Durchdringungskante (z. B. $s_{1}=s_{i}$ ) mit schwacher Kopplung rechnen, d. h. die Differenz $w_{1}-w_{i}=\sqrt{u^{2}+1} \approx u$ setzen. Diese Differenz ist ja für den Streifenverlauf in der Umgebung von $s_{1}=s_{i}$ maßgebend. Natürlich gelten diese Überlegungen genau so für die Umgebung jeder beliebigen Durchdringungskante $s_{j}=s_{k}$, die ja ebenso durch einen Gittervektor $\mathfrak{g}=\mathfrak{h}_{i}-\mathfrak{h}_{k}$ und eine Strukturamplitude $\mathrm{S}_{\mathrm{g}}=\mathrm{S}_{j k}$ gekennzeichnet ist. Die Näherung $\sqrt{u^{2}+1} \approx u$ bedeutet für $\boldsymbol{u}=1$ einen Fehler von $30 \%$, für $\boldsymbol{u}=\mathbf{2}$ schon nur noch einen Fehler von $10 \%$. Die Einheit von $u$ ist aber nach (11), wenn man die Strukturamplitude $\left|S_{i 1}\right|=k^{2}\left|s_{i 1}\right|$ durch die entsprechende $\left|U_{i 1}\right|=\frac{\hbar^{2}}{2 m}\left|S_{i 1}\right|$ der Schwankung der potentiellen Energie des Elektrons ersetzt:

$$
e_{i}=\frac{2 m}{\hbar^{2}} \frac{1}{\left|\mathfrak{h}_{i}{ }^{\mathrm{t}}\right|^{2}}\left|\frac{d}{k} \mathfrak{h}_{i}^{\mathrm{t}}\right|\left|U_{i 1}\right|
$$


Für Muskovit gibt MacGillavry ${ }^{3}\left|U_{331}\right|=2,4 \mathrm{eV}$ und von $\mathrm{La} \mathrm{ue}^{1}(1 / 2 \pi)\left|\mathfrak{h}_{331}\right|=0,668 \AA-1$, damit wird

$e_{331} /\left|(d / k) \mathfrak{h}_{331}\right| \approx 3,6 \cdot 10^{-2} \approx e_{060} /\left|(d / k) \mathfrak{h}_{060}\right|$

Das bedeutet auf den üblichen Aufnahmen (z. B. $\left.\left|(d / k) \mathfrak{h}_{331}\right|=3 \mathrm{~cm}\right)$ etwa $1 \mathrm{~mm}$ als Einheit. Die übrigen Strukturamplituden von Muskovit scheinen alle kleiner zu sein, die zu hoch indizierten Vektoren im reziproken Gitter gehörigen Einheiten werden außerdem noch wegen des Faktors $\left|\mathrm{ht}^{\mathrm{t}}\right|$ im Nenner von $e_{i}$ kleiner. Man kann wohl annehmen, daß die durch die vorliegende Theorie nicht erfaßten Umgebungen von Punkten mit Kopplungen höherer Ordnung etwa dieselbe Größenordnung haben.

Bei den von $\mathrm{F}$ u es ${ }^{2}$ behandelten Beispielen liegt $c_{i}$ zwischen 1,4 und 2,6, so daß für obiges Beispiel der asymptotische Streifenabstand $\pi / c_{i}$ zwischen 1,2 und $2,2 \mathrm{~mm}$ betragen würde.

Bei dieser Betrachtungsweise darf man keinesfalls übersehen, daß die Interferenzstreifen minimaler Intensität auf den Aufnahmen wegen ihrer natürlichen Unschärfe nicht beliebig genau auszumessen sind. Für einen Vergleich mit dem Experiment würde deshalb eine wesentliche Erhöhung der Genauigkeit der Theorie gar keinen merklichen Gewinn bedeuten.

Wir haben festgestellt, daß die Bereiche, in denen Doppel- und Umweganregungen eintreten können, bei Glimmer nur sehr schmale Streifen auf dem Film bilden. Kopplungen höherer Ordnung treten immer dort auf, wo sich solche Streifen überschneiden. Es tritt nun die Frage auf, ob diese „Kopplungsstreifen“ nicht in so großer Zahl auftreten, daß sie trotz ihrer geringen Breite die ganze Aufnahme überdecken. Das würde bedeuten, daß die von uns gemachte Voraussetzung ,starke Kopplung von gleichzeitig nicht mehr als zwei Strahlen" praktisch überhaupt nicht zu erfüllen wäre.

Betrachtet man z. B. Aufnahmen mit symmetrischer Einstrahlung [Abb. 19, 20, 21 bei Kossel und Möllenstedt ${ }^{4}$, Abb. 11 bei Möllenstedt ${ }^{5}$, die den von $\mathrm{F}$ u e s ${ }^{2}$ durchgeführten Rechnungen zugrunde liegen, oder auch die Abb. 15 bei Kos s el und Möllenstedt ${ }^{4}$ und die Abb. 6, 7, 8 bei Möllenstedt ${ }^{5}$, so fallen auf diesen eine Vielzahl von Reflexen auf, die von $\mathrm{F}$ u e s ${ }^{6}$ als eine „Art aus

3 C. H. Mac Gill a vry, Physica 7, 329 [1940].

4 W. Kossel u. G. Möllenstedt, Ann. Physik (5) 36, 113 [1939].

5 G. M ölle n st e d t, Ann. Physik (5) 40, 39 [1941].
Kikuchi-Linien zusammengesetzter D e b y e S cherrer-Ringe“ charakterisiert wurden. Diese „Ringreflexe" rühren offensichtlich alle von Netzebenen her, die sich für bestimmte Einfallsrichtungen aus dem Primärstrahl in B ragg scher Reflexionsstellung befinden. Sie stören also alle die "großen“ Reflexe 060, 331 usw. durch Doppelanregung. Die Intensität dieser B r a g g schen Reflexe ist von gleicher Größenordnung wie die des Primärstrahls [das folgt schon aus der einfachen Formel (1) des Zweistrahlfalls], während auf den symmetrischen Aufnahmen die Intensität der "großen Reflexe“ selbst $\simeq s^{2}$ und damit klein gegen die des Primärstrahls ist. Wir haben also bei unseren Rechnungen nicht etwa alle „starken Strahlen“ (im Sinne von großer Intensität) der betreffenden Aufnahme berücksichtigt. (Und die angekündigte weitere Arbeit wird an einer Reihe von Beispielen zeigen, daß es darauf gar nicht ankommt.) Trotzdem können mit der vorliegenden Theorie auch solche Einzelheiten der Streifenstruktur wie die Streifenverbiegungen quantitativ gedeutet werden.

Es ist zwar einzusehen, daß die erwähnten schmalen Reflexe keine Streifenverbiegungen hervorrufen können. Dazu braucht nur die betreffende Strukturamplitude so klein zu séin, daß das zugehörige $c_{k}$ unter dem ersten kritischen Wert liegt. Dies gilt auch für die Vielzahl der nicht in Erscheinung tretenden Umweganregungen, deren Zahl von gleicher Größenordnung sein muß wie die der Doppelanregungen. Nicht einzusehen ist jedoch, warum sich der große Intensitätsverlust des Primärstrahls (die Zahl der Ringreflexe liegt auf einzelnen Aufnahmen zwischen 10 und 100) durch diese Reflexe nicht irgendwie im Verlauf der Interferenzstreifen äußert.

Man muß deshalb annehmen, daß man die Wechselwirkung des Primärstrahls mit allen übrigen stark gekoppelten Strahlen für die Gesamtheit seiner Einfallsrichtungen in guter Näherung summarisch als Schwächung des Primärstrahls mit einem von der Einfallsrichtung unabhängigen, konstanten Faktor berücksichtigen darf, wenn man die Intensität eines bestimmten Flecks berechnen will.

6 E. Fues, Ann. Physik (5) 43, 538 [1943]. 